\title{
Lattice Boltzmann solver of Rossler equation
}

\author{
Guangwu YAN*,** and Li RUAN* \\ * LSEC, Institute of Computational Mathematics, Academy of Mathematics and System Sciences, \\ Chinese Academy of Sciences, Beijing 100080, China \\ ** Department of Mathematics, Jilin University, Changchun 130023, China
}

(Received April 10, 2000)

\begin{abstract}
We proposed a lattice Boltzmann model for the Rossler equation. Using a method of multiscales in the lattice Boltzmann model, we get the diffusion reaction as a special case. If the diffusion effect disappeared, we can obtain the lattice Boltzmann solution of the Rossler equation on the mesescopic scale. The numerical results show the method can be used to simulate Rossler equation.
\end{abstract}

Keywords: lattice Boltzmann method, multiscale technique, Rossler equation

\section{Introduction}

Nonlinear ordinary differential equations have a widely physical background and practical role. Not only in the area of dynamical systems, but also on other physical fields, we have much interest on its chaotic behaviour which can be used to explore and comprehend complicated phenomena. In this paper, we construct a lattice Boltzmann method to Rossler equations

$$
\begin{aligned}
& \frac{\mathrm{d} u}{\mathrm{~d} t}=-(v+w) \\
& \frac{\mathrm{d} v}{\mathrm{~d} t}=u+A v \\
& \frac{\mathrm{d} w}{\mathrm{~d} t}=B+w(u-C)
\end{aligned}
$$

explained and structured the Rossler equation ${ }^{[1,2]}$, and give a new numerical technique. In recent years, the lattice Boltzmann method has attracted attention as an alternative numerical scheme for simulation of fluid flows ${ }^{[3-5]}$. The main idea of the lattice Boltzmann methods is to get available macroscopic physical equations by using the BGK type Boltzmann equation. In the general case, time, space and velocity are discrete on one lattice, and then, choosing the equilibrium distribution function to fit some requirements which can be obtained with multiscale technique and Chapman-Enskog expansion.

The Rossler equation is a simple ordinary differential equation. It can be seen as a diffusion reaction equation by reducing the diffusion effect if we assume the macroscopic varies have the form $u=u(t), v=v(t), w=w(t)$. It is easy to construct the diffusion reaction equation by using the lattice Boltzmann model. As a special case, the equilibrium distribution function will be simple. In this paper, we construct the lattice Boltzmann model of the Rossler equation, simulate the phase space pictures and plot the bifurcation profile. The numerical results are good enough.

\section{The lattice Boltzmann model}

We discrete the velocity of particles into $b$ directions in $D$-dimensional space, the lattice with unit spacing is used where each node has $b$ nearest neighbors connected by $b$ links. The distribution function $f_{\alpha}^{\sigma}$ is the probability of finding a particle at time $t$, node $x$, with velocity $e_{\alpha}$, where $\alpha=0,1, \cdots, b$ ( $\alpha=0$ is the particle at rest). For one-dimensional lattice, $D=1$, 
$b=2$, the particles velocity are $e_{\alpha}=(0, c,-c)$; for two-dimensional lattice, $D=2, b=4$, leads to $2 D$ squarc modcls. The macroscopic quantity is defined by

and the conservation condition is

$$
u^{\sigma}=\sum_{\alpha} f_{\alpha}^{\sigma}(\boldsymbol{x}, t)
$$

$$
\sum_{\alpha} f_{\alpha}^{\sigma, \mathrm{eq}}(\mathbf{x}, t)=u^{\sigma}(\boldsymbol{x}, t)
$$

where $f_{\alpha}^{\sigma, \text { eq }}$ and $u^{\sigma}$ are the equilibrium distribution function and the density of the species $\sigma$ at time $t$ and position $x$ respectively, $\sigma=1,2, \cdots, M, M$ is the number of species.

The lattice Boltzmann equation reads

$$
f_{\alpha}^{\sigma}(\boldsymbol{x}+\Delta \boldsymbol{x}, t+\Delta t)-f_{\alpha}^{\sigma}(\boldsymbol{x}, t)=-\frac{1}{\tau}\left[f_{\alpha}^{\sigma}(\boldsymbol{x}, t)-f_{\alpha}^{\sigma, \mathrm{eq}}(\boldsymbol{x}, t)\right]+\omega_{\alpha}^{\sigma}(\boldsymbol{x}, t)
$$

where $\omega_{\alpha}^{\sigma}$ is the additional term caused by chemical reactions, $\tau$ is the single relaxation time factor $^{[6]}$. In the case of standard lattice Boltzmann ${ }^{[3-5]}$, the term $\omega_{\alpha}^{\sigma}=0$.

Select the small parameter $\varepsilon$ as the time unit in numerical simulation, which can also play the role of the Knudsen number ${ }^{[5]}$. The lattice Boltzmann equation in physical units is

$$
f_{\alpha}^{\sigma}\left(\boldsymbol{x}+\varepsilon \boldsymbol{e}_{\alpha}, t+\varepsilon\right)-f_{\alpha}^{\sigma}(\boldsymbol{x}, t)=-\frac{1}{\tau}\left[f_{\alpha}^{\sigma}-f_{\alpha}^{\sigma, \mathrm{eq}}\right]+\omega_{\alpha}^{\sigma}
$$

Applying the Chapman-Enskog expansion ${ }^{[6]}$ to $f_{\alpha}^{\sigma}$ under the assumption that mean free path is of the same order of $\varepsilon$, and expanding $f_{\alpha}^{\sigma}$ about $f_{\alpha}^{\sigma,(0)}=f_{\alpha}^{\sigma, \text { eq }}$, we get

$$
f_{\alpha}=f_{\alpha}^{\sigma,(0)}+\varepsilon f_{\alpha}^{\sigma,(1)}+\varepsilon^{2} f_{\alpha}^{\sigma,(2)}+\varepsilon^{3} f_{\alpha}^{\sigma,(3)}+\varepsilon^{4} f_{\alpha}^{\sigma,(4)}+\cdots
$$

Using the multiscale technique, we introduce the different time scales

thus

$$
t_{0}=t, \quad t_{1}=\varepsilon t, \quad t_{2}=\varepsilon^{2} t, \quad t_{3}=\varepsilon^{3} t, \quad \cdots
$$

$$
\frac{\partial}{\partial t}=\frac{\partial}{\partial t_{0}}+\varepsilon \frac{\partial}{\partial t_{1}}+\varepsilon^{2} \frac{\partial}{\partial t_{2}}+\varepsilon^{3} \frac{\partial}{\partial t_{3}}+O\left(\varepsilon^{4}\right)
$$

To discuss changes in different time scales, we get a series of lattice Boltzmann equations in different time scales ${ }^{[7]}$

and set

$$
\frac{\partial f_{\alpha}^{\sigma,(0)}}{\partial t_{0}}+e_{\alpha} \frac{\partial f_{\alpha}^{\sigma,(0)}}{\partial \boldsymbol{x}}=-\frac{1}{\tau} f_{\alpha}^{\sigma,(1)}
$$

$$
\omega_{\alpha}^{\sigma}=\varepsilon^{2} \phi_{\alpha}^{\sigma}
$$

Eq. (4) is an important assumption, its meaning is reaction effect and diffusion effect at the same time scale $\varepsilon^{2}$. Taking into assumption (4), we have

and

$$
\frac{\partial f_{\alpha}^{\sigma,(0)}}{\partial t_{1}}-\tau\left(1-\frac{1}{2 \tau}\right)\left(\frac{\partial}{\partial t_{0}}+e_{\alpha} \frac{\partial}{\partial x}\right)^{2} f_{\alpha}^{\sigma,(0)}=-\frac{1}{\tau} f_{\alpha}^{\sigma,(2)}+\phi_{\alpha}^{\sigma}
$$

$$
\begin{gathered}
\frac{\partial f_{\alpha}^{\sigma,(0)}}{\partial t_{2}}+(1-2 \tau)\left(\frac{\partial}{\partial t_{0}}+\boldsymbol{e}_{\alpha} \frac{\partial}{\partial x}\right) \frac{\partial f_{\alpha}^{\sigma,(0)}}{\partial t_{1}}+\left(\tau^{2}-\tau+\frac{1}{6}\right)\left(\frac{\partial}{\partial t_{0}}+e_{\alpha} \frac{\partial}{\partial x}\right)^{3} f_{\alpha}^{\sigma,(0)} \\
=-\frac{1}{\tau} f_{\alpha}^{\sigma,(3)}+(-\tau)\left(\frac{\partial}{\partial t_{0}}+e_{\alpha} \frac{\partial}{\partial x}\right) \phi^{\sigma}\left(u^{\sigma}\right)
\end{gathered}
$$

Taking $(3)+(5) \times \varepsilon$, and the summation about $\alpha$, we obtain

$$
\frac{\partial u^{\sigma}}{\partial t}=\lambda^{\sigma} \varepsilon\left(\tau-\frac{1}{2}\right) \nabla^{2} u^{\sigma}+(b+1) \varepsilon \phi^{\sigma}\left(u^{\sigma}\right)+O\left(\varepsilon^{2}\right)
$$


Choose

$$
\begin{gathered}
\sum_{\alpha} f_{\alpha}^{\sigma,(0)} e_{\alpha}=0 \\
\sum_{\alpha} f_{\alpha}^{\sigma,(0)} e_{\alpha i} e_{\alpha j}=\lambda^{\sigma} u^{\sigma} \delta_{i j}
\end{gathered}
$$

where $\lambda^{\sigma}=\xi^{\sigma} / \varepsilon(\tau-1 / 2)$ are parameters in the equilibrium distribution function to control the diffusivity. Combining (6) and (8), we get

$$
\frac{\partial u^{\sigma}}{\partial t}=\xi^{\sigma} \frac{\partial^{2} u^{\sigma}}{\partial x_{i} \partial x_{i}}+(b+1) \varepsilon \phi^{\sigma}\left(u^{\sigma}\right)+O\left(\varepsilon^{2}\right)
$$

If $(b+1) \varepsilon \phi^{\sigma}\left(u^{\sigma}\right)=\psi^{\sigma}\left(u^{\sigma}\right)$ as source term of Eq. (9), we get the truncation error $R=O\left(\varepsilon^{2}\right)$. From Eqs. (2), (7) and (8), the equilibrium distribution function $f_{\alpha}^{\sigma,(0)}$ can be expressed as follows

$$
f_{\alpha}^{\sigma,(0)}=\left\{\begin{array}{l}
u^{\sigma}-\frac{\lambda^{\sigma} D u^{\sigma}}{c^{2}}, \quad \alpha=0 \\
\frac{\lambda^{\sigma} D u^{\sigma}}{b c^{2}}, \quad \alpha=1, \cdots, b
\end{array}\right.
$$

From Eq. (9), we obtain the following diffusion-reaction equations $\left(M=3,\{u\}^{\sigma}=(u, v, w)\right)$ :

$$
\begin{aligned}
& \frac{\partial u}{\partial t}=\xi^{1} \nabla^{2} u-(v+w) \\
& \frac{\partial v}{\partial t}=\xi^{2} \nabla^{2} v+u+A v \\
& \frac{\partial w}{\partial t}=\xi^{3} \nabla^{2} w+B+w(u-C)
\end{aligned}
$$

where $A, B, C$ are parameters in the Rossler equations. Let the parameter $\lambda^{\sigma} \rightarrow 0$ and assume the space is uniform, then $u=u(t), v=v(t), w=w(t)$. Eqs. (11) turn into the Rossler equations (1).

We select two cases of $\lambda^{\sigma}$ : (1) $\lambda^{\sigma}=0.0$ corresponding Eqs. (1). In this case, the equilibrium distribution functions is simple

$$
f_{\alpha}^{\sigma}=\left\{\begin{array}{l}
u^{\sigma}, \quad \alpha=0 \\
0, \quad \alpha=1, \cdots, b
\end{array}\right.
$$

(2) $\lambda^{\sigma}=1.0 \times 10^{-3}$, corresponding Eqs. (11). In this case, the equilibrium distribution functions are Eqs. (10).

For numerical simulation, we set a one-dimensional region $[0,1]$, with initial conditions

$$
u^{\sigma}=u_{0}^{\sigma}, \quad t=0
$$

and boundary conditions

$$
\frac{\partial u^{\sigma}(0, t)}{\partial x}=\frac{\partial u^{\sigma}(1, t)}{\partial x}=0
$$

\section{Numerical tests}

The Rossler Equation describes a nonlinear chemical reaction system. It possesses chaotic phenomenon when the parameter $C \geq 5.0$. In this paper, we select the parameters as $A=0.20$, $B=0.20$ and $C=5.0$ for the reaction terms, the parameters in the lattice Boltzmann equation being $c=10.0,1 / \tau=2.2, b=2, \lambda^{\sigma}=1.0 \times 10^{-3}$. From Eq. (9), the additional term is given as follows 


$$
\begin{gathered}
\phi^{1}=\frac{1}{\varepsilon(b+1)}(-v+w), \quad \phi^{2}=\frac{1}{\varepsilon(b+1)}(u+A v), \quad \phi^{3}=\frac{1}{\varepsilon(b+1)}[B+w(u-C)] \\
\lambda^{1}=\frac{\xi^{1}}{\varepsilon(\tau-1 / 2)}, \quad \lambda^{2}=\frac{\xi^{2}}{\varepsilon(\tau-1 / 2)}, \quad \lambda^{3}=\frac{\xi^{3}}{\varepsilon(\tau-1 / 2)}
\end{gathered}
$$

Fig. 1 shows the results calculated with parameters $1 / \tau=2.2, c=10.0$. It is seen that the Rossler attractor appear. Note the equation is partial differential equation with diffusion in this model, and using a little lattice (100 lattices). The numerical result shows the phase picture with attractor, which agrees with theoretical analysis. We also plot the bifurcation graph using parameter $C$ and the maximum $u_{\max }$ of $u$ in the 20000 iterative steps (Fig. 1d).

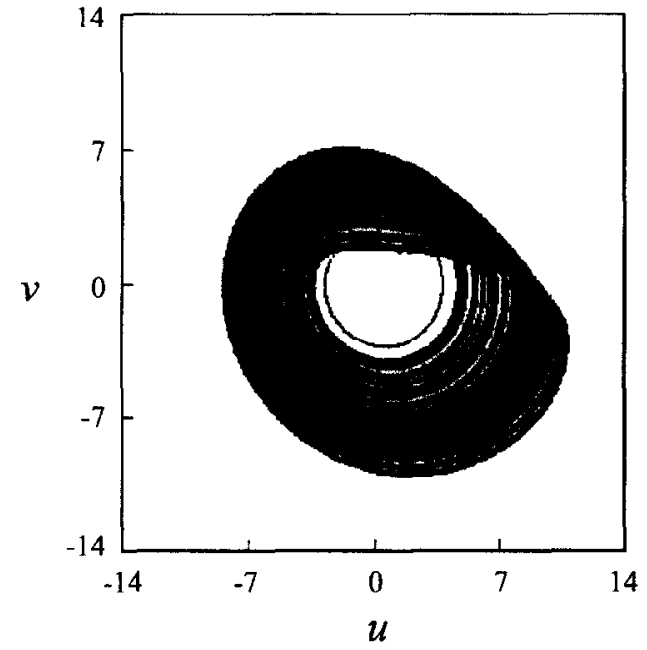

(a)

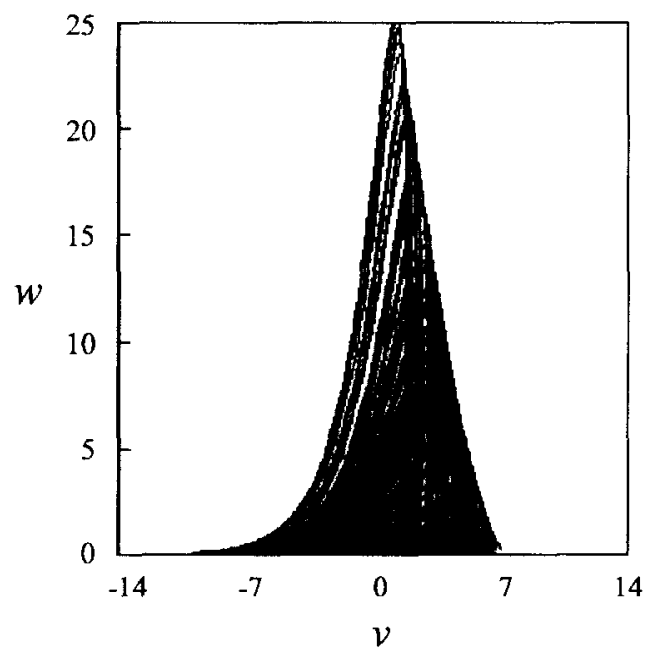

(c)

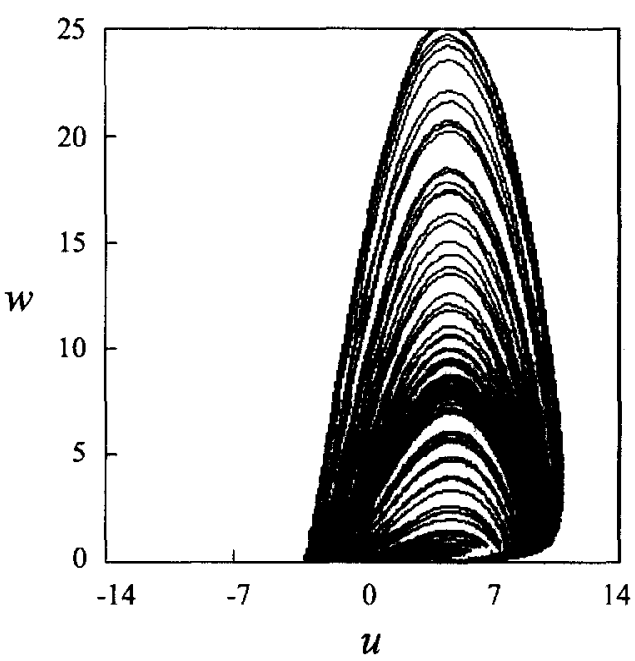

(b)

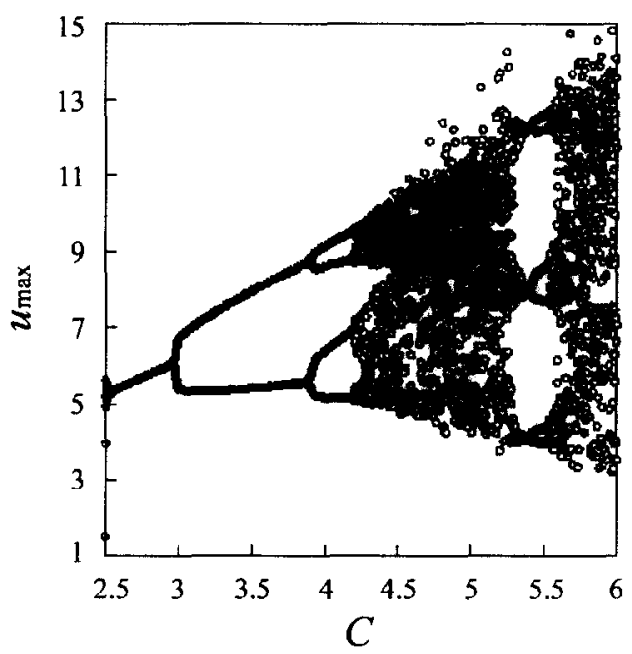

(d)

Fig. 1 (a)-(c) The phase pictures of Rossler equation with parameters $A=0.20, B=0.20$ and $C=5.0, \xi^{1}=\xi^{2}=\xi^{3}=1.0 \times 10^{-3}$, (d) the bifurcation picture of $u_{\max }$ versus $C$ 


\section{Conclusion}

In this paper, we presented a lattice Boltzmann method for solving the Rossler equation. We obtained the lattice Boltzmann solution of Rossler equation from $\xi^{\sigma}>0$. The result in $D_{\sigma}>0$ may be inspiring, which gives a new method for the Rossler attractor. It is easy to construct other nonlinear chemical reaction systems by using higher moments method of lattice Boltzmann method. As a numerical method, it may be considered as a tool to get patterns of some chaos and chemical reactions. We use assumption (4) in this model. This assumption is very important to construct the model, the meaning of this assumption is that the reaction and diffusion have same order scales.

\section{Acknowledgment}

This work is supported by the National Key Program for Developing Basic Sciences (No. G1999032801).

\section{References}

[1] Rossler, O. E., Chaotic bchaviour in simple reaction system, Z. Naturf., 1976, 31a: 259-264

[2] Rossler, O. E., An equation for continuous chaos, Phys. Lett. A, 1976, 57: 397-398

[3] Qian, Y. H., d'Humieres, D. and Lallemand, P., Lattice BGK model for Navier-Stokes equation, Europhys. Lett., 1992, 17, 6: 479-484

[4] Chen, H. D., Chen, S. Y. and Matthaeus, M. H., Recovery of the Navier-Stokes equations using a lattice Boltzmann method, Phys. Rev. A, 1992, 45: 5339-5342

[5] Benzi, R., Succi, S. and Vergassola, M., The lattice Boltzmann equations: Theory and applications, Phys. Reports, 1992, 222: 147-197

[6] Dawson, P., Chen, S. Y. and Doolen, G. D. et al., Lattice molthed and their applications to reacting systems, J. Chem. Phys., 1993, 98(2): 1514-1523

[7] Yan Guangwu, Chen Yaosong and Hu Shouxin, Simple lattice Boltzmann method for simulating flows with shock wave, Phys. Rev. E, 1999, 59: 454-459 